\title{
An Intercomparison between ERA-Interim Reanalysis and Observed Precipitation in Northeast China
}

\author{
Jie Zhou ${ }^{1}$ and Junhu Zhao ${ }^{2}$ \\ ${ }^{1}$ Yongchuan Meteorological Bureau of Chongqing Province, Chongqing 402160, China \\ ${ }^{2}$ Laboratory for Climate Studies, National Climate Center, China Meteorological Administration, Beijing 100081, China
}

Correspondence should be addressed to Jie Zhou; zhoujie1226@126.com

Received 29 July 2015; Accepted 25 October 2015

Academic Editor: Yong-Ping Wu

Copyright (C) 2015 J. Zhou and J. Zhao. This is an open access article distributed under the Creative Commons Attribution License, which permits unrestricted use, distribution, and reproduction in any medium, provided the original work is properly cited.

\begin{abstract}
Recently, the European Center for Medium-Range Weather Forecasts (ECMWF) released a new set of reanalysis data-ERAInterim. We make an intercomparison between ERA-Interim precipitation and observed precipitation in Northeast China. The results show that, in general, the ERA-Interim reanalysis precipitation data can describe the spatial and temporal characteristics of seasonal precipitation in Northeast China well. In terms of spatial distribution, ERA-Interim precipitation is generally consistent with the observation data in different seasons in Northeast China. There is a larger difference in the center of Northeast China than in other areas between the two kinds of data. The ERA-Interim precipitation is larger than observed precipitation in most of Northeast China. In spring, autumn, and winter, the ERA-Interim precipitation value is close to the observation one, while in summer there is a large difference in Liaoning Peninsula and Changbai Mountain between the two kinds of precipitation data. In terms of temporal characteristics, the time series of the ERA-Interim precipitation matches well with the observed precipitation in whole. In different seasons, the annual variation of the ERA-Interim precipitation is well correlated with that of the observed precipitation.
\end{abstract}

\section{Introduction}

Northeast China is regarded as one of the largest commodity grain bases and most potential agriculture production areas in China. Summer is not only the main season of crops growing, but also the season of precipitation concentration. The amount of precipitation and its distribution are important factors affecting grain production in Northeast China. On account of its location in middle-high latitudes of the Eurasian continent, the precipitation over the region is influenced by the East Asian Summer Monsoon (see [1-3]) and the circulation in the middle-high latitude (see $[4,5]$ ) jointly. The combined effects of multiscale circulation systems lead to the frequent occurrence of climatic disasters such as floods and droughts in summer. In recent years, the significant increase of droughts and floods has been shown to cause great loss to the local economy, especially the agricultural production (see [6]). Therefore, it has important scientific significance to study the temporal and spatial variation of water cycle and the relationship of water vapor budget and balance in Northeast China.

From different perspectives, the meteorological scholars have studied the water cycle in Northeast China. Cui Yuqin (see [7]) analyzed the moisture input and output of different boundaries by using the data from 18 air sounding stations and found that the moisture input was larger than the output around the whole year in this region. Sun (see [8]) and Gao (see [9]), respectively, used station data and NCEP reanalysis data to analyze the spatial distribution of integrated precipitable water vapor in Northeast China and found that the precipitation appeared on a diminishing scale from south to north and from east to west in this region.

Recently, the European Center for Medium-Range Weather Forecasts (ECMWF) provides a set of more comprehensive medium-term reanalysis data, the ERA-Interim data (see $[10,11])$. However, the credibility and quality of the ERAInterim reanalysis data in Northeast China have not been compared and analyzed. Thus, this paper makes a comparison 
between the ERA-Interim reanalysis precipitation and the observed one in Northeast China by adopting some objective analytic methods. In this way, we expect to reveal specific differences between the two kinds of data, to evaluate the credibility of ERA-Interim precipitation over Northeast China and furtherly to make a preparation for the calculation of the characteristics of all elements and the water budget in a comprehensive, systematic, and quantificational way, which can provide more accurate reference information for summer precipitation predictions based on our existing one (see [12-21]).

\section{Data and Methods}

2.1. Data. The data used in this paper include the following.

(1) ERA-Interim reanalysis monthly average precipitation (see [10]) supplied by ECMWF, with horizontal resolution of $2.5 \times 2.5$. Northeast region mentioned in this paper includes Heilongjiang, Jilin, and Liaoning provinces and the region east to $115^{\circ} \mathrm{E}$ of Inner Mongolia. The period lasted from January 1979 to December 2010.

(2) Daily precipitation data of 756 stations provided by the China Meteorological Data Sharing Service System. Monthly average precipitation is derived from daily data. By excluding some stations, of which the time sequence of precipitation is too short or there are too many missing values, 95 stations of Northeast China are selected.

\subsection{Methods}

2.2.1. Interpolating Grid Data to Stations. The bilinear interpolation method is used to interpolate grid data to stations. The core idea of this method is to carry out a linear interpolation in two directions, respectively. Monthly average ERA-Interim precipitation of 95 stations in Northeast China can be acquired through this method.

Suppose that $P$ is the station where we want to get the ERA-Interim precipitation. Around station $P$, there are four grids: $P_{11}, P_{21}, P_{12}$, and $P_{22} . P_{11}$ and $P_{21}$ have the same latitude which is the closest to station $P$ among stations whose latitude is lower than that of station $P$. Similarly, $P_{12}$ and $P_{22}$ have the same latitude which is the closest to station $P$ among stations whose latitude is higher than that of station $P . P_{11}$ and $P_{12}$ have the same longitude which is the closest to station $P$ among stations whose latitude is lower than that of station $P . P_{21}$ and $P_{22}$ have the same longitude which is the closest to station $P$ among stations whose latitude is higher than that of station $P$. The precipitation values and the latitude $(\theta)$ and longitude $(\lambda)$ of the four grids are all known: $P_{11}\left(\theta_{1}, \lambda_{1}\right)$, $P_{21}\left(\theta_{1}, \lambda_{2}\right), P_{12}\left(\theta_{2}, \lambda_{1}\right)$, and $P_{22}\left(\theta_{2}, \lambda_{2}\right)$. The latitude $(\theta)$ and longitude $(\lambda)$ of station $P$ are $\theta_{0}$ and $\lambda_{0}$, respectively. By using the bilinear interpolation, we can get the ERA-Interim precipitation of station $P$ from the precipitation of the four grids. Specific formulas are as follows.
First, linear interpolation is performed in the zonal direction:

$$
\begin{aligned}
& R_{1}=\frac{\lambda_{2}-\lambda_{0}}{\lambda_{2}-\lambda_{1}} P_{11}+\frac{\lambda_{0}-\lambda_{1}}{\lambda_{2}-\lambda_{1}} P_{21} \\
& R_{2}=\frac{\lambda_{2}-\lambda_{0}}{\lambda_{2}-\lambda_{1}} P_{12}+\frac{\lambda_{0}-\lambda_{1}}{\lambda_{2}-\lambda_{1}} P_{22}
\end{aligned}
$$

In (1), $R_{1}$ is the precipitation at the grid which has the same latitude with $P_{11}$ and $P_{21}$ and the same longitude with station $P$. In the same way, in (2), $R_{2}$ is the precipitation at the grid which has the same latitude with $P_{12}$ and $P_{22}$ and the same longitude with station $P . P_{11}, P_{21}, P_{12}$, and $P_{22}$ denote the values of precipitation at the four girds, respectively.

Then, linear interpolation is performed in the meridional direction:

$$
P=\frac{\theta_{2}-\theta_{0}}{\theta_{2}-\theta_{1}} R_{1}+\frac{\theta_{0}-\theta_{1}}{\theta_{2}-\theta_{1}} R_{2} .
$$

So, the ERA-Interim precipitation of station $P$ is calculated as follows:

$$
\begin{aligned}
P= & \frac{P_{11}}{\left(\lambda_{2}-\lambda_{1}\right)\left(\theta_{2}-\theta_{1}\right)}\left(\lambda_{2}-\lambda_{0}\right)\left(\theta_{2}-\theta_{0}\right) \\
& +\frac{P_{21}}{\left(\lambda_{2}-\lambda_{1}\right)\left(\theta_{2}-\theta_{1}\right)}\left(\lambda_{0}-\lambda_{1}\right)\left(\theta_{2}-\theta_{0}\right) \\
& +\frac{P_{12}}{\left(\lambda_{2}-\lambda_{1}\right)\left(\theta_{2}-\theta_{1}\right)}\left(\lambda_{2}-\lambda_{0}\right)\left(\theta_{0}-\theta_{1}\right) \\
& +\frac{P_{22}}{\left(\lambda_{2}-\lambda_{1}\right)\left(\theta_{2}-\theta_{1}\right)}\left(\lambda_{0}-\lambda_{1}\right)\left(\theta_{0}-\theta_{1}\right) .
\end{aligned}
$$

2.2.2. Interpolating Observed Data to Grids. Cressman objective analysis function is an interpolation method for progressive revisions to interpolate discrete points into the regular grid ones within small errors, which is widely used in kinds of diagnostic analysis and objective analysis of numerical forecast (see [22]). This approach is first proposed by Cressman (see [23]): the first guess field is given and then the observed field is used to gradually correct the first guess one until the revised field approximates observational record. The mathematical formula is

$$
a^{\prime}=a_{0}+\Delta a_{i j}
$$

where

$$
\Delta a_{i j}=\frac{\sum_{k=1}^{K}\left(W_{i j k}^{2} \Delta a_{k}\right)}{\sum_{k=1}^{K} W_{i j k}} .
$$

In (5) and (6), $a$ donates any meteorological element, herein as precipitation; $a_{0}$ represents the first guess of variable $a$ on $(i, j) ; a^{\prime}$ is the revised value of variable $a$ on $(i, j) ; \Delta a_{k}$ shows the difference between the observed value at observation point $k$ and the first guess one; $W_{i j k}$ is a weighting factor 
TABLE 1: Similarity coefficients of spatial distribution of ERAInterim and observed precipitation in different seasons over Northeast China.

\begin{tabular}{lccc}
\hline Spring & Summer & Autumn & Winter \\
\hline 0.985 & 0.992 & 0.986 & 0.969 \\
\hline
\end{tabular}

changing between 0.0 and $1.0 ; K$ means the number of stations within the influence radius $R$. The weight function $W_{i j k}$ is crucial to Cressman objective analysis, with general form:

$$
W_{i j k}= \begin{cases}\frac{R^{2}-d_{i j k}^{2}}{R^{2}+d_{i j k}^{2}}, & d_{i j k}<R \\ 0, & d_{i j k} \geq R .\end{cases}
$$

In (7), selection of radius of influence $R$ is impacted by human factor to some extent, and usually the constant is the first choice. The commonly used radii of influence are 1,2, 4, 7 , and 10 . They would be chosen as radius of influence in turn. $d_{i j k}$ denotes the distance between $(i, j)$ and the observation point $k$.

\section{Interpolating Grid Data to Stations}

In order to analyze and evaluate the specific differences between ERA-Interim reanalysis precipitation and the observed one, firstly, this paper uses bilinear interpolation method to get ERA-Interim precipitation (hereinafter station ERA-Interim precipitation) of 95 stations in Northeast China through interpolation grid precipitation to stations, corresponding results shown in Figure 1. The left shows ERA-Interim precipitation in four seasons of 95 stations in Northeast China, while the right is the observed one.

As shown in Figure 1, in spring and winter, the spatial distribution of ERA-Interim precipitation is consistent with that of the observed one, with the fact that precipitation centers in Changbai Mountain area, while less occurs in central Northeast China and northern Inner Mongolia. In contradiction to sparse precipitation isopleths in the plain area, Changbai Mountain area has intensive ones. The conditions in summer and autumn are similar to that in spring and winter, but the large value area of ERA-Interim precipitation is more northern than that of the observed one. In general, in the same season, the two sets of data present similar spatial distribution, with the occurrence of ERA-Interim precipitation larger than the observed one.

In order to quantitatively characterize the degree of similarity of the spatial distribution between ERA-Interim and observed precipitation in different seasons, this paper calculates the spatial similarity coefficient (see [24]) of ERAInterim and observed precipitation (Table 1). It can be seen from Table 1 that the spatial similarity coefficients between ERA-Interim and observed precipitation in four seasons all exceed 0.95 . The maximum one appears in the summer, reaching 0.992 , and the minimum one in winter, reaching 0.969 . This indicates that the spatial distribution of precipitation in Northeast China in four seasons can be well simulated by the ERA-Interim reanalysis data, especially in summer.
TABLE 2: Correlation coefficients of time series of ERA-Interim and observed precipitation in different seasons.

\begin{tabular}{lccc}
\hline Spring & Summer & Autumn & Winter \\
\hline 0.942 & 0.930 & 0.937 & 0.783 \\
\hline
\end{tabular}

To quantitatively characterize the specific differences between ERA-Interim and observed precipitation of 95 stations through bilinear interpolation, this paper compares ERA-Interim precipitation to the observed one (observed precipitation minus ERA-Interim precipitation). And results are shown in Figure 2. The solid blue dots represent negative values, while the solid red triangles indicate positive values. The bigger (smaller) the solid dot (triangle) is, the greater (smaller) the difference is. In spring, the value of ERAInterim precipitation is bigger than that of observed precipitation in most part of the area except for Liaodong Peninsula. Small differences lie in the center of the Northeast, while great ones in surrounding area, especially in Inner Mongolia and south Heilongjiang, greatest difference reaching around $50 \mathrm{~mm}$.

In summer, the greatest differences between ERA-Interim precipitation and the observed one mainly lie in the southern area. The biggest positive values exist in Liaodong Peninsula, while the biggest negative ones are around Changbai Mountains. And the maximum magnitude of positive and negative difference both reached $150 \mathrm{~mm}$. The difference distribution between the two kinds of precipitation data in autumn is in line with that in spring. In winter, the ERAInterim precipitation is little bigger than the observed one over the whole region. And the difference between them is the smallest among four seasons, only about $26 \mathrm{~mm}$. Conclusions can be made that, in terms of values of the precipitation, ERA-Interim ones are greater than the observed ones in the four seasons, where ERA-Interim simulates the spatial distribution of observed precipitation the best in winter, with small differences in the whole region, and the worst in summer, with the two kinds of precipitation differing significantly in the southern region of Northeast China.

For the comparison of the time sequence between ERAInterim and observation data, ERA-Interim grid values of Northeast China are interpolated into stations to get the average time sequence of 95 stations (Figure 3), and the correlation coefficient (Table 2) between the two time series is calculated. The solid black dots in Figure 3 denote ERA-Interim precipitation, while the red ones represent the observed precipitation. In conjunction with Figure 3 and Table 2, as for the same season, the area averaged ERA-Interim precipitation is larger than the observed one in different seasons.

In different seasons, the correlation coefficients of the two time series all have passed the 0.01 confidence level. In spring, summer, and autumn, the correlation coefficients are close to each other, ranging from 0.93 to 0.94 , while in winter it takes 0.78 as the minimum. All these show that ERA-Interim precipitation simulates annual variability of the seasonal station precipitation in Northeast China fairly well. 

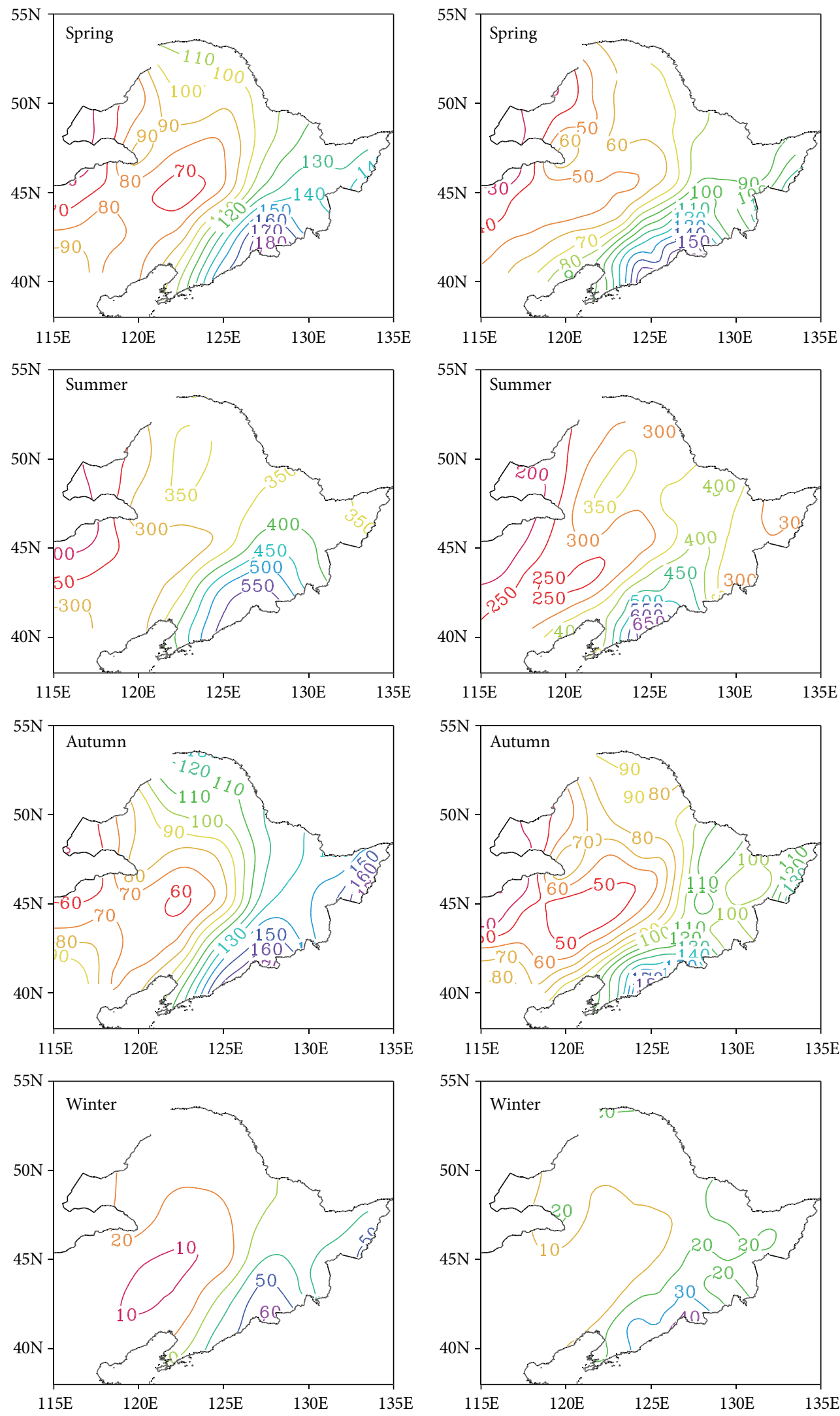

(a)

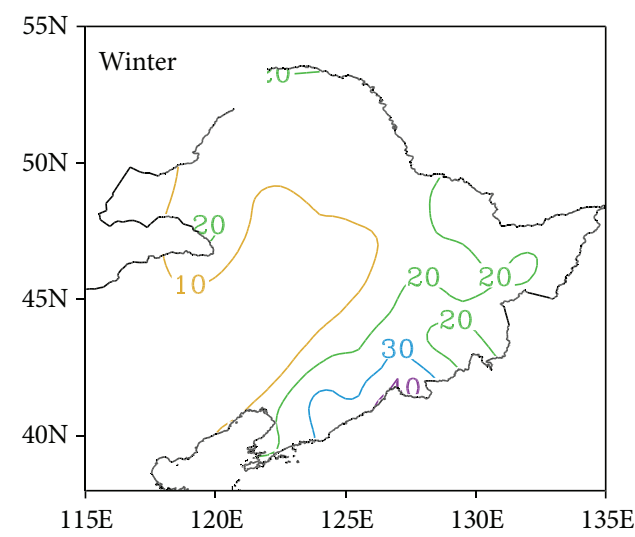

(b)

FIgURE 1: Spatial distribution of ERA-Interim (a) and observed (b) precipitation in different seasons over Northeast China (mm). 

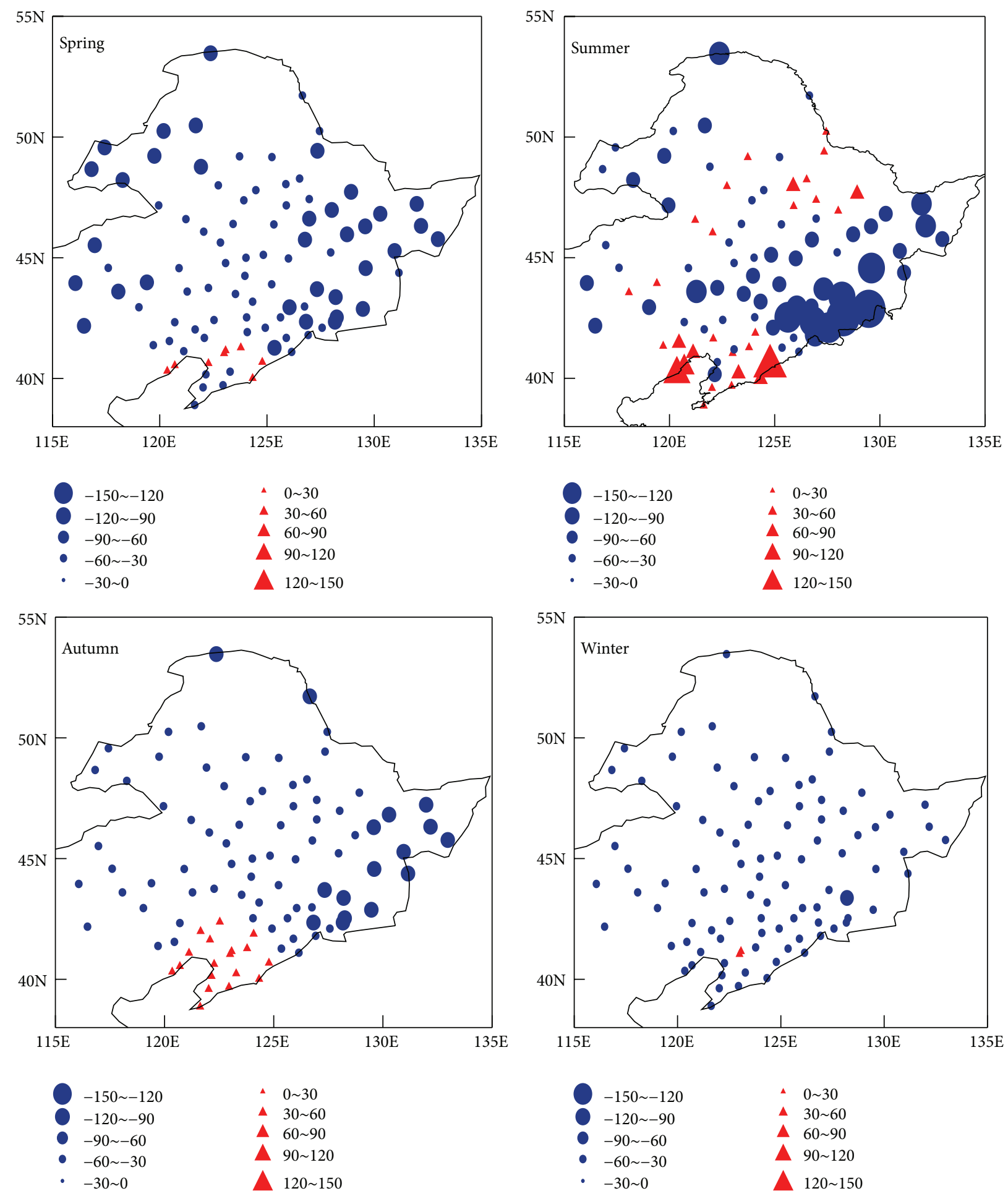

$\triangle \quad 0 \sim 30$

\ $30 \sim 60$

- $60 \sim 90$

А $90 \sim 120$

120 150

FIGURE 2: Spatial distribution of the difference between observed precipitation and ERA-Interim precipitation in four seasons in Northeast China (unit: $\mathrm{mm}$ ). 


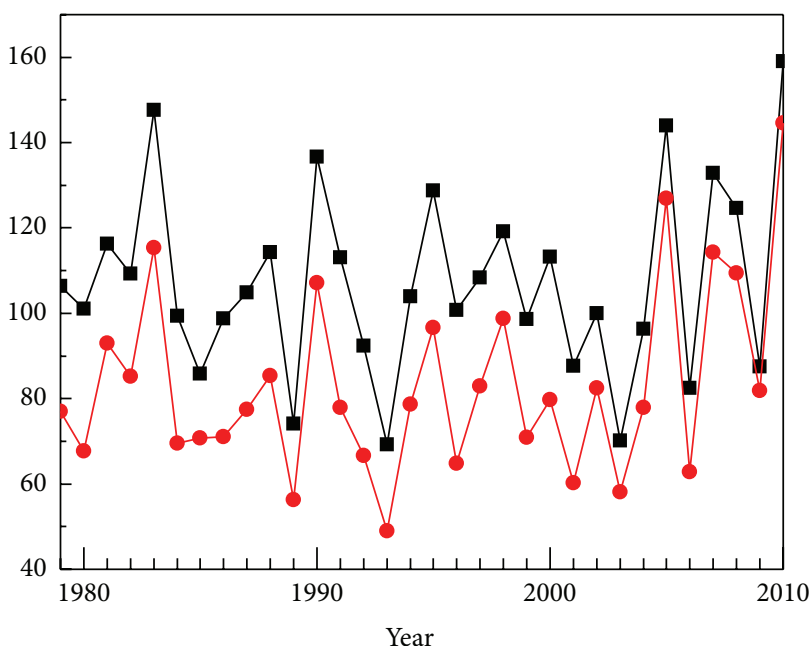

(a) Spring

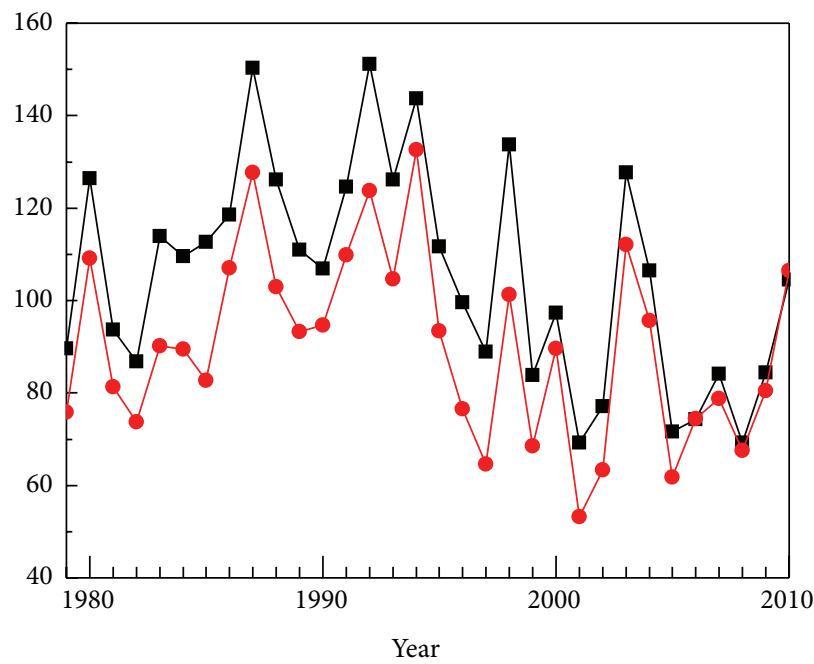

(c) Autumn

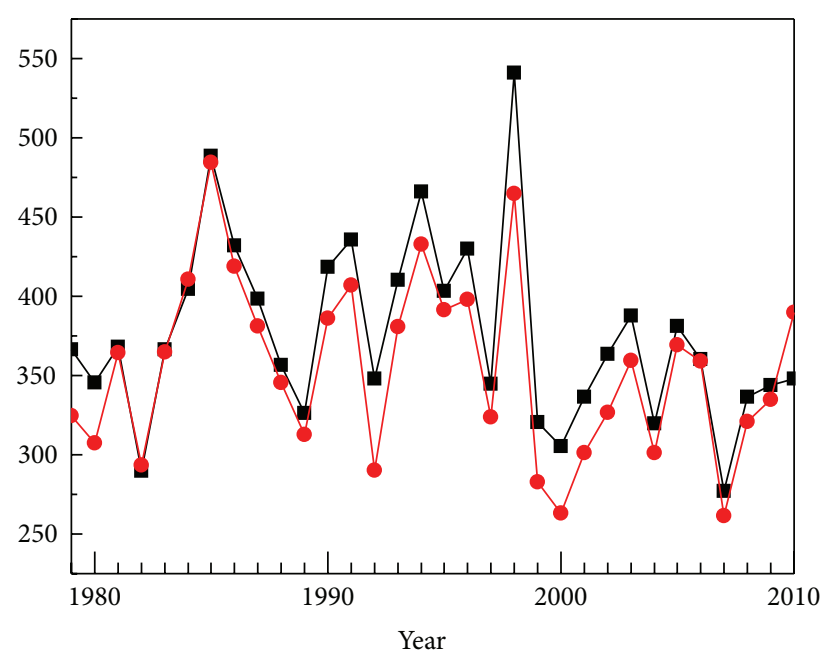

(b) Summer

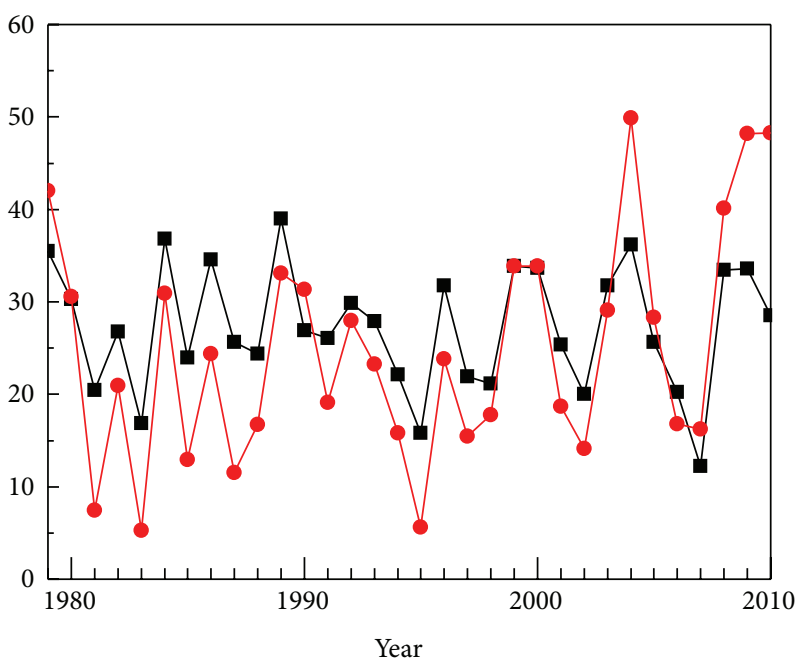

(d) Winter

FIGURE 3: Time series of ERA-Interim and observed precipitation (unit: $\mathrm{mm}$ ) (solid black dots denote ERA-Interim precipitation, while red ones represent the observed precipitation).

And in different seasons, the ERA-Interim precipitation and the station precipitation generally have a significant positive correlation relationship. ERA-Interim precipitation is slightly greater than the observed one in four seasons.

\section{Interpolating Station Data into Grids}

In the last section, bilinear interpolation is employed to get the ERA-Interim values of seasonal precipitation of 95 stations in Northeast China. And the comparison is made between the ERA-Interim and observed precipitation in spatial distribution and temporal variation separately. For the spatial distribution, ERA-Interim precipitation in different seasons is greater than the observed one in entire Northeast China, characterized by small differences in central region and great ones in surrounding area; the smallest differences appear in winter. For the temporal variation, ERA-Interim precipitation is always greater than observed one in the four seasons.

To verify the objectivity and rationality of the above results, the Cressman objective analysis function is used to interpolate the station precipitation into grid points to get the new grid precipitation data. Then compassion is made between the new grid precipitation and ERA-Interim one in spatial distribution and temporal variation in different seasons.

Figure 4 is the difference of spatial distribution of the new grid precipitation and ERA-Interim precipitation in Northeast China. As can be seen from Figure 4, ERA-Interim precipitation is greater than the new grid one in entire Northeast China in all seasons. The differences between them are small in central region of Northeast China and great in surrounding area. And the smallest differences appear in winter. The similarity coefficients between the new grid precipitation and ERA-Interim one (Table 3) are significantly high in different 

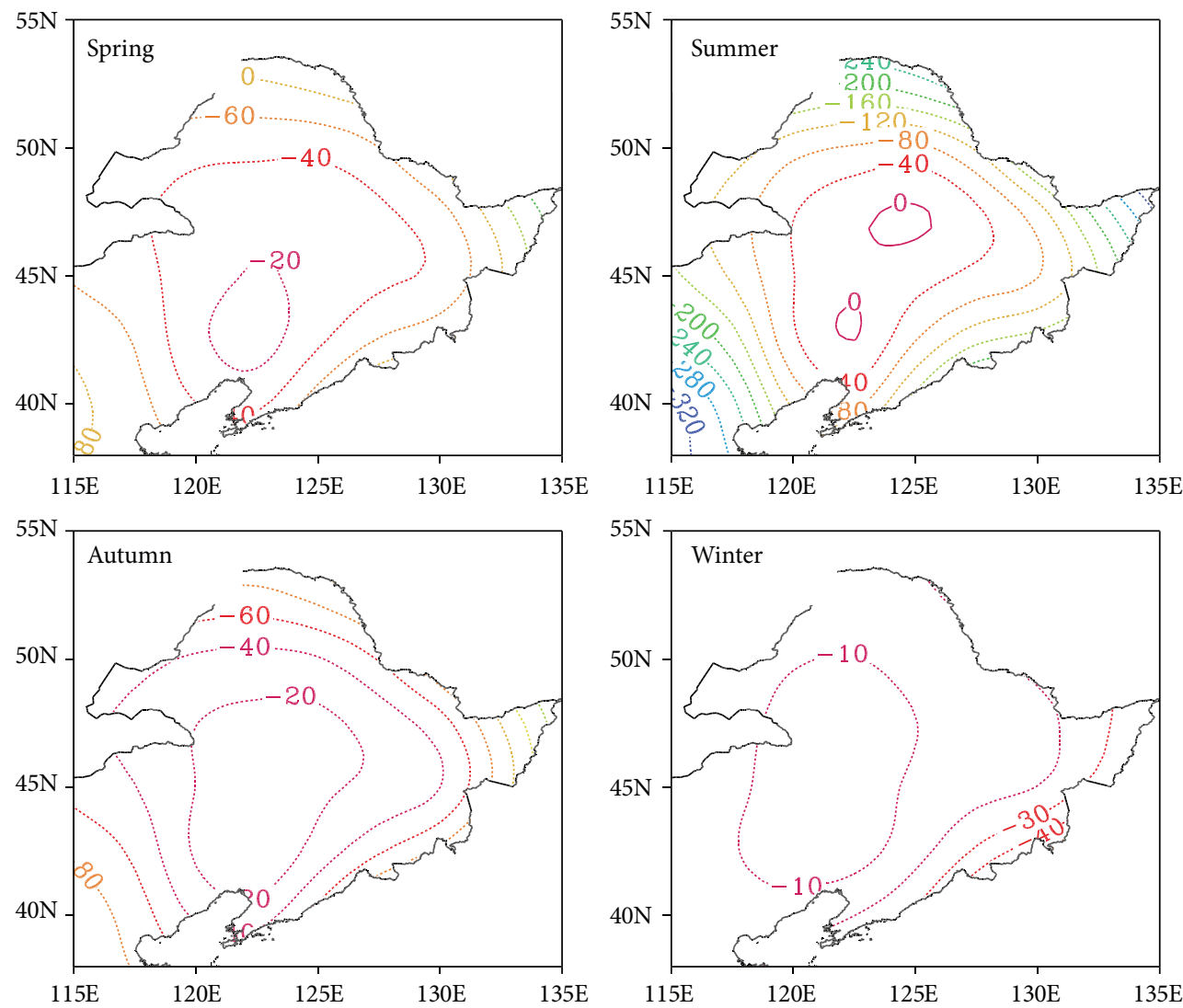

FIGURE 4: Difference of the new grid precipitation and ERA-Interim precipitation in different seasons (unit: $\mathrm{mm}$ ).

TABLE 3: Similarity coefficients of spatial distribution of the new grid precipitation and the ERA-Interim precipitation in different seasons.

\begin{tabular}{lccc}
\hline Spring & Summer & Autumn & Winter \\
\hline 0.871 & 0.883 & 0.858 & 0.878 \\
\hline
\end{tabular}

seasons, reaching over 0.85 , which indicates that the spatial distributions of them are very similar to each other. The most similar season is summer, and the least one is autumn.

The difference of the spatial distribution of the two kinds of grid data has been analyzed above. The difference in temporal variation of them is shown in Figure 5. The solid black dots denote ERA-Interim precipitation, while the red ones represent the new grid precipitation. As can be seen from Figure 5, the average ERA-Interim precipitation in the area is greater than the new grid one around the whole year. The feature of their annual variation is almost the same. The correlation coefficients between them (Table 4) have passed the 0.01 confidence level, which implies that there is a significant positive correlation between them. In spring, summer, and autumn, the correlation coefficients are close to each other, ranging from 0.89 to 0.91 , while in winter it takes 0.82 as the minimum. Through the analysis and comparison above, we can see that the result of Cressman objective analysis function is roughly the same with that of the bilinear interpolation.
TABlE 4: Correlation coefficients of time series of the new grid precipitation and the ERA-Interim precipitation in different seasons.

\begin{tabular}{lccc}
\hline Spring & Summer & Autumn & Winter \\
\hline 0.917 & 0.895 & 0.900 & 0.825 \\
\hline
\end{tabular}

\section{Conclusions and Discussion}

By adopting the bilinear interpolation and the Cressman objective analysis function, the differences of the two kinds of precipitation data in spatial distribution and temporal variation have been made a comparison. And the following conclusions can be reached:

(1) For the spatial distribution, the spatial pattern of ERA-Interim precipitation in different seasons is roughly consistent with that of the observation field, reflecting small differences in central Northeast China, and great ones in surrounding area. As for entire Northeast China, ERA-Interim precipitation in most areas is more than the observed one. In spring, autumn, and winter, the values of the two kinds of precipitation are closer to each other, while in summer there is a big difference in Liaoning Peninsula and Changbai Mountains.

(2) For the temporal variation, ERA-Interim data simulates the annual variability of observed precipitation 


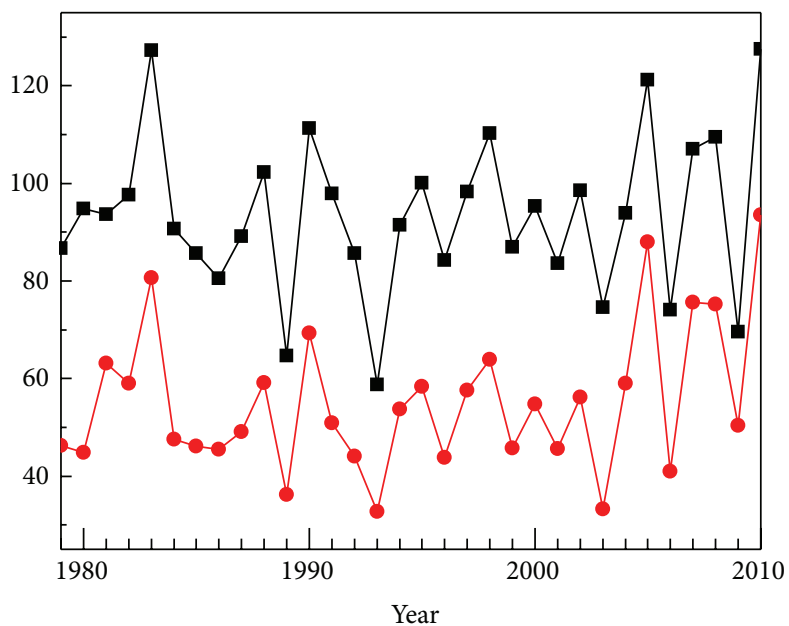

(a) Spring

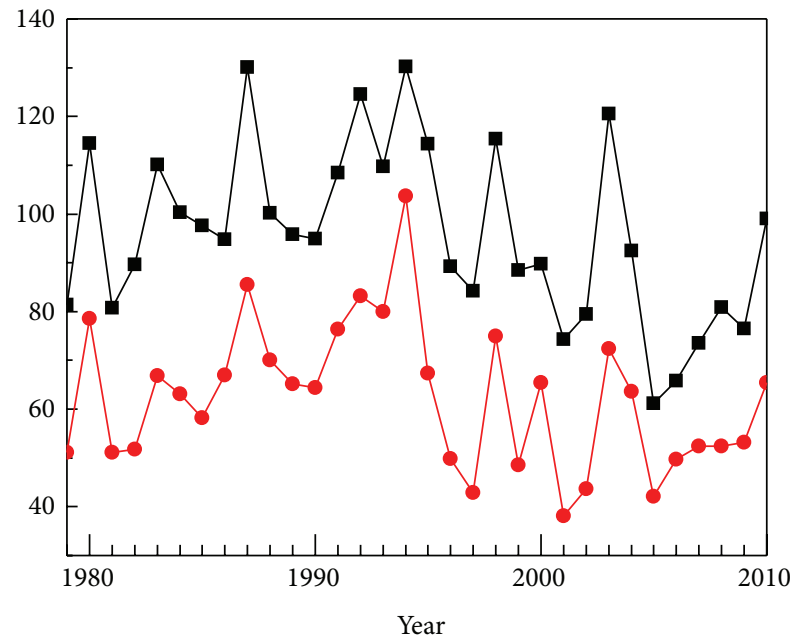

(c) Autumn

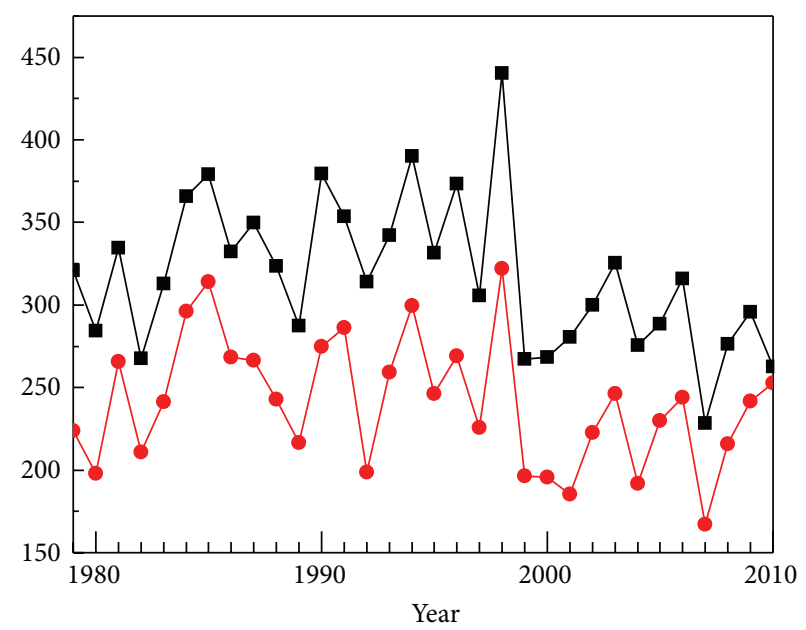

(b) Summer

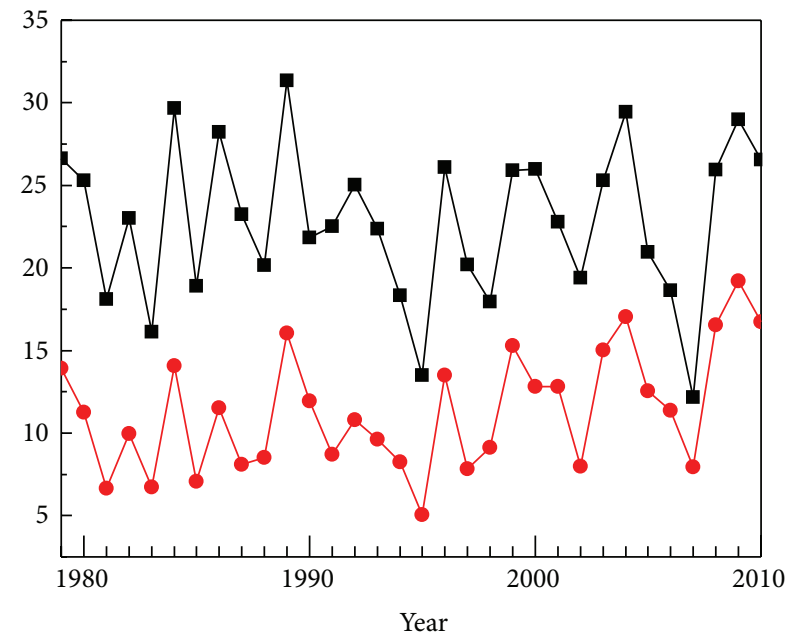

(d) Winter

FIGURE 5: Time series of the new gird precipitation and ERA-Interim precipitation in different seasons (unit: mm) (solid black dots denote ERA-Interim precipitation, while red ones represent the new grid precipitation).

in Northeast China fairly well. In the four seasons, there are all significant positive correlation between the ERA-Interim precipitation and the observed one. And ERA-Interim precipitation is slightly greater than the observed one.

(3) In general, ERA-Interim reanalysis precipitation can simulate the variation of spatial distribution and time series of the observed precipitation in four seasons in Northeast China fairly well.

Northeast China has complex terrain, resulting in a significant impact on precipitation. This paper fails to consider the impact of the terrain during the comparison and analysis of the ERA-Interim and observation data. In the future, if the terrain factors are successfully taken into account in the duration of any analysis and comparison of the differences of the two data, as to seek a better interpolation method, ERAInterim reanalysis may be evaluated more objectively.

\section{Conflict of Interests}

The authors declare that there is no conflict of interests regarding the publication of this paper.

\section{Acknowledgment}

This work is supported by the National Natural Science Foundation of China (Grant nos. 41530531, 41375079, and 41505061).

\section{References}

[1] Y. Lian, B.-Z. Shen, Z.-T. Gao, G. An, and X.-L. Tang, "The study of the on-set criterion and the date of East Asian summer monsoon in Northeast China and its main characteristic analysis," Acta Meteorologica Sinica, vol. 61, no. 5, pp. 548-559, 2003. 
[2] Y. Lian, B.-Z. Shen, and Z.-T. Gao, "An exploration on the determination of east Asia summer monsoon index," Acta Mechanica Sinica, vol. 62, no. 6, pp. 782-789, 2004.

[3] B. Z. Shen, Z. D. Lin, R. Y. Lu, and Y. Lian, "Circulation anomalies associated with interannual variation of early- and late-summer precipitation in Northeast China," Science China Earth Sciences, vol. 54, no. 7, pp. 1095-1104, 2011.

[4] J.-H. He, Z.-W. Wu, L. Qi, and A.-J. Jiang, "Relationships among the Northern Hemisphere annual mode, the Northeast Cold vortex and the summer rainfall in Northeast China," Journal of Meteorology and Environment, vol. 22, no. 1, pp. 1-5, 2006.

[5] B.-Y. Wu, R.-H. Zhang, and D.-A. Rosanne, "Arctic dipole anomaly and summer precipitation in Northeast China," Chinese Science Bulletin, vol. 53, no. 12, pp. 1422-1428, 2008.

[6] Y. Lian, Z.-T. Gao, B.-Z. Shen, H.-L. Ren, X.-L. Tang, and Y.Y. Liu, "Climate change and its impacts on grain production in Jinlin province," Advances in Climate Change Research, vol. 3, no. 1, pp. 46-49, 2007.

[7] Y.-Q. Cui, "Budget condition of water vapor over northeast China and its source," Scientia Geographica Sinica, vol. 15, no. 1, pp. 80-87, 1995.

[8] L. Sun, "Spatial and temporal variation of atmospheric watervapor resources over northeast China," Scientia Geographica Sinica, vol. 27, no. 1, pp. 2-9, 2007.

[9] Z.-T. Gao, "Moisture transportation and budget over Northeast China," Scientia Geographica Sinica, vol. 27, no. zl, pp. 28-38, 2007.

[10] D. P. Dee, S. M. Uppala, A. J. Simmons et al., "The ERA-Interim reanalysis: configuration and performance of the data assimilation system," Quarterly Journal of the Royal Meteorological Society, vol. 137, no. 656, pp. 553-597, 2011.

[11] P. Berrisford, P. Kållberg, S. Kobayashi et al., "Atmospheric conservation properties in ERA-Interim," Quarterly Journal of the Royal Meteorological Society, vol. 137, no. 659, pp. 1381-1399, 2011.

[12] J.-P. Huang and S.-W. Wang, "The experiments of seasonal prediction using the analogy-dynamical model," Science in China Series B: Chemistry, vol. 35, no. 2, pp. 207-216, 1992.

[13] J.-P. Huang, Y.-H. Yi, S.-W. Wang, and J.-F. Chou, "An analoguedynamical long-range numerical weather prediction system incorporating historical evolution," Quarterly Journal-Royal Meteorological Society, vol. 119, no. 511, pp. 547-565, 1993.

[14] J. Yang, J.-H. Zhao, Z.-H. Zheng, K.-G. Xiong, and B.-Z. Shen, "Estimating the prediction errors of dynamical climate model on the basis of prophase key factors in north China," Chinese Journal of Atmospheric Sciences, vol. 36, no. 1, pp. 11-22, 2012.

[15] J. Yang, Q.-G. Wang, R. Zhi, and G.-L. Feng, "Dynamic optimal multi-indexes configuration for estimating the prediction errors of dynamical climate model in North China," Acta Physica Sinica, vol. 60, no. 2, Article ID 029204, pp. 827-839, 2011.

[16] K.-G. Xiong, J.-H. Zhao, G.-L. Feng, J.-P. Huang, and J.-G. $\mathrm{Hu}$, "A new method of analogue-dynamical prediction of monsoon precipitation based on analogue prediction principal components of model errors," Acta Physica Sinica, vol. 61, no. 14, pp. 551-561, 2012.

[17] K.-G. Xiong, G.-L. Feng, J.-P. Huang, and J.-F. Chou, "Analoguedynamical prediction of monsoon precipitation in northeast china based on dynamic and optimal configuration of multiple predictors," Acta Meteorologica Sinica, vol. 25, no. 3, pp. 316-326, 2011.
[18] Q.-G. Wang, G.-L. Feng, Z.-H. Zheng, and R. Zhi, "A study of the objective and quantifiable forecasting based on optimal factors combinations in precipitation in the middle and lower reaches of the Yangtze River in summer," Chinese Journal of Atmospheric Sciences, vol. 35, no. 2, pp. 287-297, 2011.

[19] Z.-H. Zheng, H.-L. Ren, and J.-P. Huang, "Analogue correction of errors based on seasonal climatic predictable components and numerical experiments," Acta Physica Sinica, vol. 58, no. 10, pp. 7359-7367, 2009.

[20] J.-H. Zhao, J. Yang, G.-L. Feng, and S.-X. Zhang, "Causes and dynamic statistical forecast of the summer rainfall anomaly over China in 2011," Quarterly Journal of Applied Meteorology, vol. 24, no. 1, pp. 43-54, 2011.

[21] J.-H. Zhao, G.-L. Feng, Q.-G. Wang, J. Yang, and Z.-Q. Gong, "Cause and prediction of summer rainfall anomaly distribution in China in 2010," Chinese Journal of Atmospheric Sciences, vol. 35, no. 6, pp. 1069-1078, 2011.

[22] Z.-X. Su and L.-S. Cheng, "The comparative study of two objective analysis methods: successive correction and optimum interpolation," Plateau Meteorology, vol. 13, no. 2, pp. 194-205, 1994.

[23] G. P. Cressman, "An operational objective analysis system," Monthly Weather Review, vol. 87, no. 10, pp. 367-374, 1959.

[24] N. Shi, Statistic Analysis and Forecast Methods in Meteorology, China Meteorological Press, Beijing, China, 2009. 


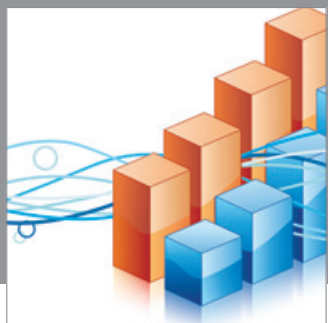

Advances in

Operations Research

mansans

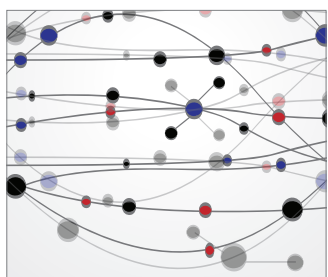

The Scientific World Journal
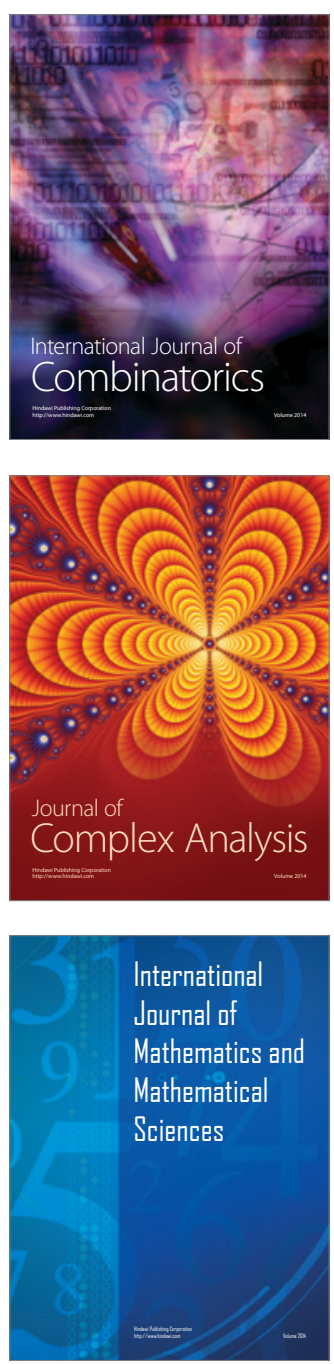
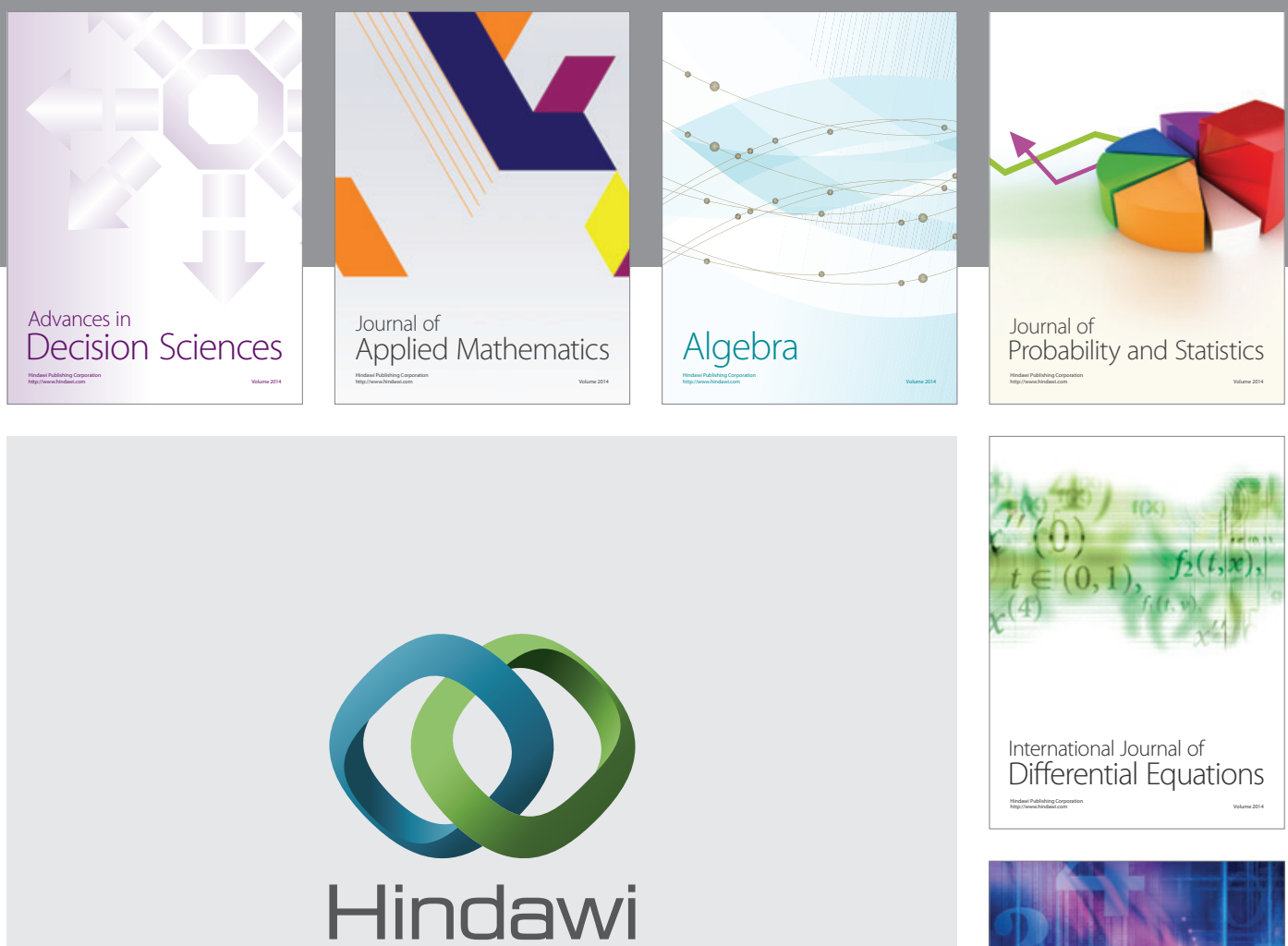

Submit your manuscripts at http://www.hindawi.com
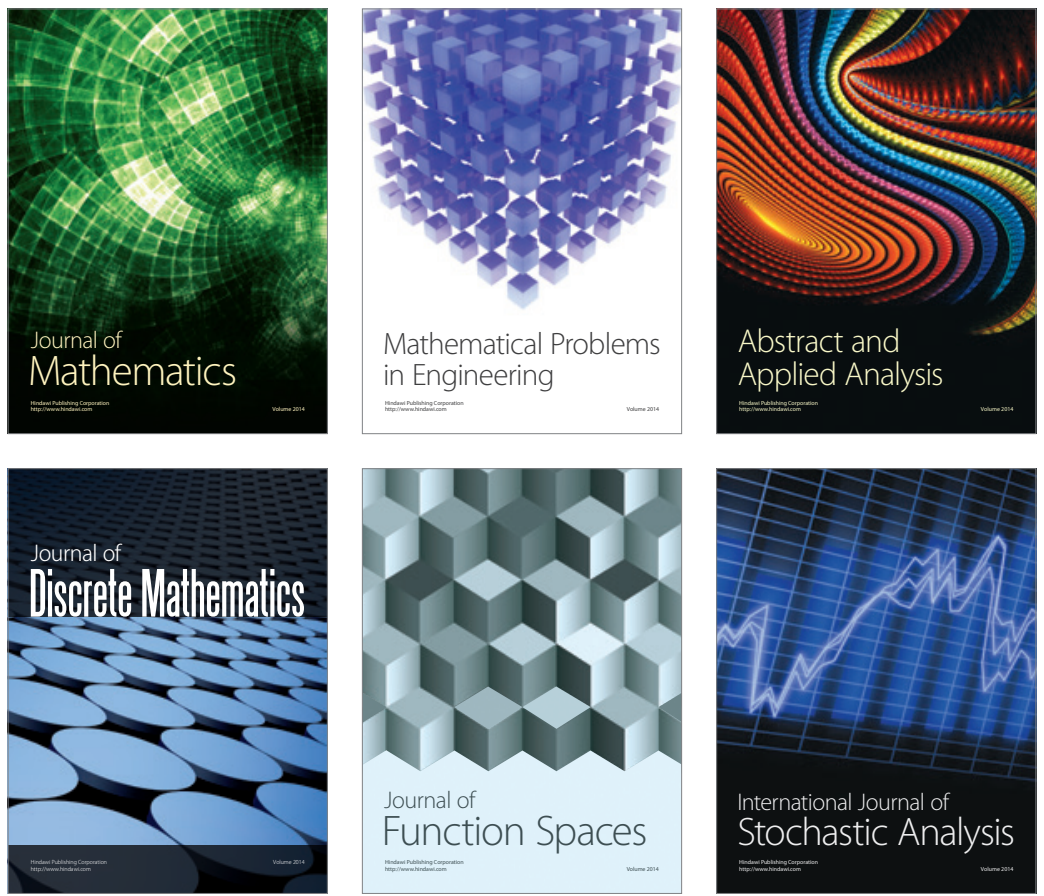

Journal of

Function Spaces

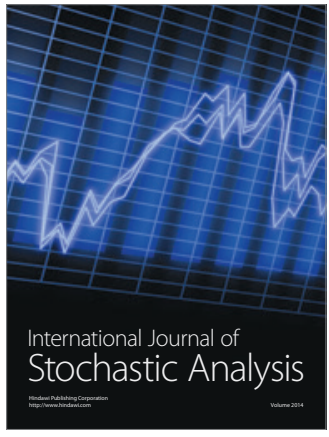

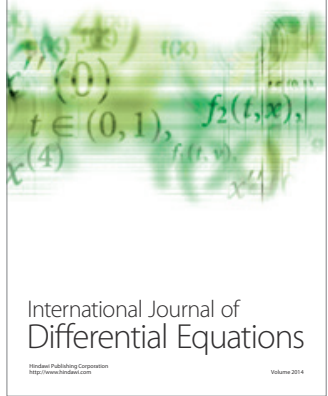
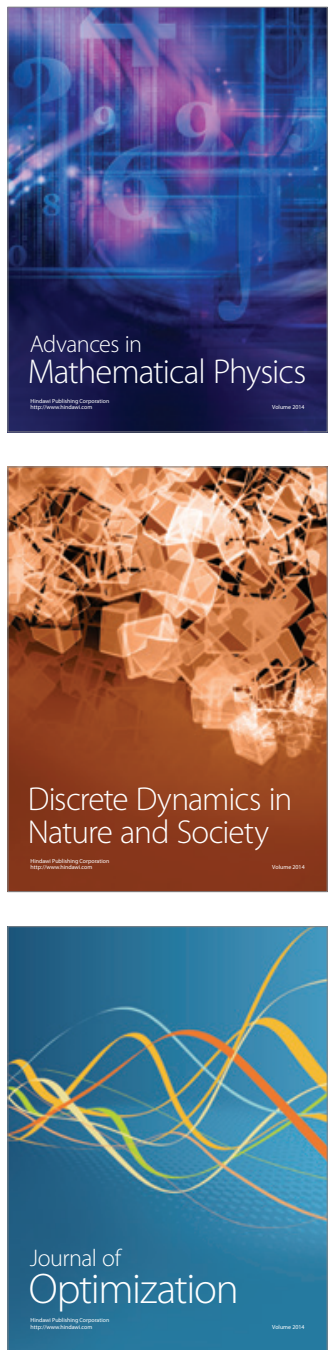